\title{
A Comparison of Human Body Compensation Models for RSSI Based Localization and Tracking
}

\author{
Jens Trogh*, David Plets*, Arno Thielens*, Luc Martens* and Wout Joseph* \\ * Department of Information Technology, iMinds - Ghent University, Belgium, jens.trogh@intec.ugent.be
}

\begin{abstract}
This preliminary work presents a comparative study of human body compensation models for localization and tracking purposes. Besides the well known influence of multipath fading and diffraction on the performance of localization systems, the influence of the user itself is often still neglected. The presence of such a user can block the radio-frequency (RF) signal paths between a body-worn node and the fixed infrastructure nodes. As a result, these additional obstructions might worsen the performance. This phenomenon is also known as human body shadowing and to cope with this effect, human body compensation models are used. In this work, compensation models obtained through electromagnetic (EM) simulations and empirical measurements are evaluated and compared.
\end{abstract}

Index Terms-Localization, Tracking, Human Body Shadowing, EM Simulation, Indoor Environment

\section{INTRODUCTION}

Localization and tracking in indoor environments has gained a huge interest over the last decade and many context-aware applications have emerged. These applications are situated in various domains, e.g., healthcare, surveillance, and in the industrial sector. The most commonly used ranging techniques for localization are angle of arrival (AoA) [1], time of arrival (ToA) [2], time difference of arrival (TDoA) [3] and received signal strength (RSS) based fingerprinting [4]. The first three are generally speaking more accurate but require specialized hardware, whereas the latter can work with the existing WiFi or ZigBee infrastructure. Many localization systems that try to cope with multipath fading and diffraction have already been proposed [5]-[7]. Although the influence of a human body has already been noted in literature [6], it is often still neglected. To this end, many localization and tracking systems are evaluated by stepwise moving a device placed on a tripod, hereby explicitly removing the human from the equation. However, practical human tracking applications, always imply the presence of a user's body. In previous work [8], two methods were proposed to cope with human body shadowing. The first method uses multiple body-worn tags and relies on the assumption that a combination of tags placed on different parts of the human body can decrease the influence of the user itself. The second method explicitly compensates for the influence of the human body by taking the user's orientation and body-worn tag's relative position into account. Next, a compensation value is calculated which is then used to preprocess the measurements before they are passed to the localization or tracking algorithm. In this work, new techniques to acquire human body compensation models are proposed and evaluated.

\section{Methodology}

In this section, the methodology to construct human body compensation models is explained in more detail.

\section{A. Simulated model}

The first model is based on simulations carried out in Semcad X, a three-dimensional full wave simulation environment based on the finite-difference time-domain (FDTD) method. The Virtual Family Male [9] (a heterogeneous phantom with a BMI $=22.3 \mathrm{~kg} / \mathrm{m}^{2}$ ) was used to perform simulations at $2.45 \mathrm{GHz}$. An accurate model of our mobile tag was built in the simulation platform and was optimized to resonate at 2.45 GHz. This mobile tag is a TelosB mote from Crossbow, equipped with an embedded PIFA antenna and Chipcon CC2420 radio. The packets broadcasted by this body worn tag are received by the fixed infrastructure and the measured RSSI values of these packets are used as input for the tracking algorithm (see Section III). In the simulation environment, the mobile tag was placed on various parts of the human phantom: chest, back, and right wrist. The simulated directivity patterns reflect the influence caused by the human body and are used to obtain three-dimensional compensation models (see Figure 1a).

\section{B. Empirical model}

The second model is based on empirical measurements carried out in a wireless testbed. This testbed measures $17 \mathrm{~m}$ by $90 \mathrm{~m}$ and is located on the third floor of an office building in Ghent. It consists of several computer labs, offices, and meeting rooms. The wireless network is a fixed infrastructure which consists of 48 nodes that are installed at a height of $2.5 \mathrm{~m}$. First, a person was asked to turn around its axis, whilst wearing a mobile tag once on the chest, back and wrist. This person turns $45^{\circ}$ every 15 seconds, taking 2 minutes for a full rotation. The mobile tags broadcast packets that are received by the fixed infrastructure, where the measured RSSI values are logged. By comparing all measured RSSI values with the average value of the full rotation, the influence of the human body can be extracted as a function of the angle (i.e., the user's orientation towards the fixed nodes). The obtained empirical compensation models for the body-worn chest, back and wrist tag are plotted in Figure 1b.

At first sight, the empirical and simulated human body compensation model show similar trends. Especially, the models 


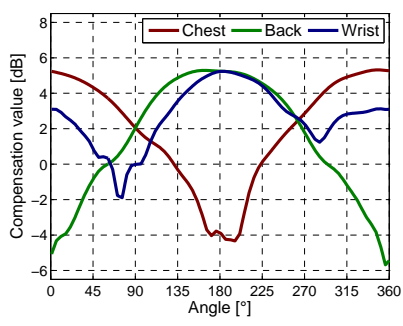

(a) Simulation

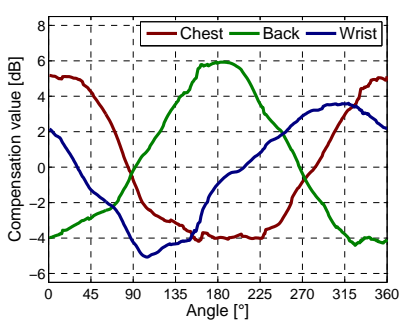

(b) Empirical measurements
Figure 1: Human body compensation models.

for the body worn tag placed on the central area of the chest and back resemble each other. The compensation models for a wrist-worn tag show more differences, this is because of the relative movement of the arm and body whilst rotating during the empirical measurements. It is also clear that a high compensation value of the chest tag corresponds to a low value of the back tag (and the other way around). This general trend can be expected as a high compensation value means the human body is blocking or attenuating the strongest path between the mobile tag and a fixed node, meaning that the tag on the other side of the user's body is likely not blocking the strongest path.

\section{TRACKING ALGORITHM}

The adopted tracking algorithm for the evaluation of the proposed compensation models is based on a Viterbi-like technique [4]. It uses a motion model and floor plan information to determine the most likely path (i.e., sequence of locations) instead of only the most likely current position (Viterbi principle). These constraints ensure that no unrealistically large distances are traveled within a given time frame and no walls are crossed. The original tracking algorithm uses measured RSSI values directly as input to calculate a location update. In this work, these measured RSSI values are preprocessed to eliminate the influence of human body shadowing. To assess the influence of a user with a certain position and orientation, the angle between their body-worn tag and the fixed infrastructure nodes, is needed as input for the compensation models of Section II. Therefore, the location of the fixed nodes (generally available in the floor plan information) and the user's orientation is utilized. The latter is provided by a compass, gyroscope or can even be estimated based on previous positions [8]. For now, this is accounted for by giving the exact orientation as an additional input. This is possible because the followed trajectory is known beforehand and it is assumed a user walks forward, hence the exact orientation can be determined.

\section{EVALUATION}

The compensation models from Section II are evaluated in terms of location accuracy. This accuracy is defined as the Euclidean distance between the predicted and actual location:

$$
\text { accuracy }=\sqrt{\left(x_{p}-x_{a}\right)^{2}+\left(y_{p}-y_{a}\right)^{2}} \quad[m]
$$

The predicted and actual positions are located at coordinates $\left(x_{p}, y_{p}\right)$ and $\left(x_{a}, y_{a}\right)$, respectively. The mean $(\mu)$, standard deviation $(\sigma)$, mean $\left(50^{\text {th }}\right.$ percentile value) and maximum $\left(95^{\text {th }}\right.$ percentile value) of the tracking accuracy are chosen as evaluation metrics. The measurement data comes from a user that walked along a test trajectory whilst wearing the mobile node once on his chest, back and wrist (repeated five times for reproducibility). The trajectory has a total length of $140 \mathrm{~m}$, passes through three meeting rooms, a computer lab, and the hallway. The mobile tags broadcast 10 packets per second which are received by the infrastructure nodes (i.e., the same testbed from Section II). Every second a location update is generated: the average RSSI values of the packets received within this second are preprocessed with the compensation models to eliminate the influence of human body shadowing and are subsequently passed to the tracking algorithm from Section III. The absolute evaluation metrics and the relative improvements compared to the original tracking algorithm (without compensation for human body shadowing), are averaged over all runs and can be found in Table I.

\begin{tabular}{l|c|c|c|c|c|c|c|c}
\hline & \multicolumn{2}{|c|}{$\mu$} & \multicolumn{2}{|c|}{$\sigma$} & \multicolumn{2}{|c|}{$50^{\text {th }}$} & \multicolumn{2}{|c}{$95^{\text {th }}$} \\
\hline Compensation $\downarrow$ & {$[\mathrm{m}]$} & {$[\%]$} & {$[\mathrm{m}]$} & {$[\%]$} & {$[\mathrm{m}]$} & {$[\%]$} & {$[\mathrm{m}]$} & {$[\%]$} \\
\hline None & 3.74 & $/$ & 2.18 & $/$ & 3.43 & $/$ & 10.25 & $/$ \\
Simulated & 3.15 & 15.78 & 1.92 & 12.25 & 2.82 & 17.78 & 9.12 & 11.03 \\
Empirical & 3.38 & 9.63 & 1.96 & 10.09 & 3.09 & 9.91 & 9.03 & 11.90 \\
\hline
\end{tabular}

Table I: Tracking accuracy and improvements with simulated and empirical compensation models.

Table I shows that all evaluation metrics benefit from the compensation of human body shadowing. The simulated compensation model slightly outperforms the empirical one but also requires more time and computing power to construct (full wave electromagnetic simulations) whereas the empirical model is based on a simple rotation whilst wearing the bodyworn tag. More specific, the mean, standard deviation, $50^{\text {th }}$ and $95^{\text {th }}$ percentile value of the tracking accuracy improve by $16 \%, 12 \%, 18 \%$, and $11 \%$ with the simulated model and $10 \%, 10 \%, 10 \%$ and $12 \%$ with the empirical compensation model. The proposed method for human body compensation adds only $8 \mathrm{~ms}$ to the processing time of one location update (on a normal desktop computer) and hence is well suited for localization or tracking in real-time.

\section{CONCLUSIONS}

In this work, human body compensation models obtained through simulations and empirical measurements were evaluated and compared. These models are used to eliminate the influence caused by human body shadowing and are designed for RSSI based localization or tracking algorithms. The simulated compensation models are based on three-dimensional electromagnetic simulations with a human phantom. Measurements in a wireless testbed with a person were used to build 
the empirical compensation models. Both models rely on the orientation of the user and the relative position of the bodyworn tag to calculate a compensation value. This compensation value is used to preprocess RSSI measurements before they are passed to the actual localization or tracking algorithm. Compensating for human body shadowing improved the median localization accuracy up to $18 \%$ with the simulated model and $10 \%$ with the empirical model. Future work will include measurements with multiple persons and other localization and tracking techniques. Furthermore, the effect of human body shadowing in different environments will be assessed.

\section{ACKNOWLEDGMENT}

This research was supported by the project IAP BESTCOM, "BElgian network on STochastic modelling, analysis, design and optimization of COMmunication systems".

\section{REFERENCES}

[1] Jun Xu, Maode Ma, and Choi Look Law. Cooperative angle-of-arrival position localization. Measurement, 59:302-313, 2015.

[2] Jie He, Yishuang Geng, Fei Liu, and Cheng Xu. Cc-kf: Enhanced toa performance in multipath and nlos indoor extreme environment. Sensors Journal, IEEE, 14(11):3766-3774, 2014.

[3] Chin-Der Wann, Yi-Jing Yeh, and Chih-Sheng Hsueh. Hybrid tdoa/aoa indoor positioning and tracking using extended kalman filters. In Vehicular Technology Conference, 2006. VTC 2006-Spring. IEEE 63rd, volume 3, pages 1058-1062. IEEE, 2006.

[4] Jens Trogh, David Plets, Luc Martens, and Wout Joseph. Advanced realtime indoor tracking based on the viterbi algorithm and semantic data. International Journal of Distributed Sensor Networks, 501:271818, 2015.

[5] Paramvir Bahl and Venkata N Padmanabhan. Radar: An in-building rfbased user location and tracking system. In INFOCOM 2000. Nineteenth Annual Joint Conference of the IEEE Computer and Communications Societies. Proceedings. IEEE, volume 2, pages 775-784. Ieee, 2000.

[6] Paramvir Bahl, Venkata N Padmanabhan, and Anand Balachandran. Enhancements to the radar user location and tracking system. Technical report, technical report, Microsoft Research, 2000.

[7] Anthea Wain Sy Au, Chen Feng, Shahrokh Valaee, Sophia Reyes, Sameh Sorour, Samuel N Markowitz, Deborah Gold, Kevaughn Gordon, and Moshe Eizenman. Indoor tracking and navigation using received signal strength and compressive sensing on a mobile device. Mobile Computing, IEEE Transactions on, 12(10):2050-2062, 2013.

[8] J. Trogh, D. Plets, A. Thielens, L. Martens, and W. Joseph. Enhanced indoor location tracking through body shadowing compensation. IEEE Sensors Journal, 16(7):2105-2114, April 2016.

[9] Andreas Christ, Wolfgang Kainz, Eckhart G Hahn, Katharina Honegger, Marcel Zefferer, Esra Neufeld, Wolfgang Rascher, Rolf Janka, Werner Bautz, Ji Chen, et al. The virtual family development of surfacebased anatomical models of two adults and two children for dosimetric simulations. Physics in medicine and biology, 55(2):N23, 2010. 